\title{
Blind Listening Evaluation of Steel String Acoustic Guitar Compensation Strategies
}

\author{
R.M. MOTTOLA ${ }^{1}$
}

Abstract-A double blind multisample intonation rating test was administered to 32 experienced guitar players/guitar builders to test perceived effectiveness of some common steel string acoustic guitar intonation compensation strategies. The test used a randomized complete block design where each treatment was a typical guitar intonation compensation strategy. Each subject completed two sequentially presented sessions. Subjects were asked to rate intonation accuracy following audition of prepared sound clips. Each clip contained a short sequence of notes recorded from steel string acoustic guitar with either perfect intonation or tuning modified to fit the intonation profile of one of three typical guitar intonation compensation strategies: straight saddle compensation, individual string saddle compensation, or individual string saddle and nut compensation. Subject ratings indicate that all compensation strategies tested were equally effective. Analysis of test results by ANOVA did not indicate significant perceived differences for either session $(p=0.596, p=0.286)$. Results of follow-up t-tests comparing intonation ratings for perfect intonation and the compensation treatment associated with the highest intonation errors (straight saddle compensation) also showed that these two treatments were equally effective in both sessions $(p=0.137, p=0.359)$. Results of follow-up Bayesian estimation analyses comparing these two treatments also indicated no discernable difference for either session (session 1 difference of means 95\% HDI: -1.31, 0.472; session 2 difference of means 95\% HDI: $-0.819,1.13)$. Subjects' correlation between ratings and actual intonation accuracy was determined by comparing ratings to intonation errors for each compensation strategy using Spearman's rank correlation. Rating correlation varied greatly among subjects $(-1 \leq \rho \leq 0.949)$. The two subjects with the best overall correlations each gave identical ratings to two different strategies. These correlations showed low correlation to subject age and to years of guitar playing experience. These analyses suggest that all compensation strategies tested are equally successful.

\section{INTRODUCTION}

Compensation is any alteration of the nominal geometric relationship among the strings and frets of a guitar or other fretted instrument intended to improve pitch accuracy of the instrument. Some conventional compensation strategies are in common use. It is possible to measure or calculate intonation errors associated with each of these strategies. Some are more accurate than others. But some of the least accurate are in widespread use and some of the most accurate are in limited use in steel string acoustic guitars. This fact suggests the possibility that effectiveness of intonation strategies may not be completely related to pitch accuracy, or that accuracy differences among common compensation strategies are small enough to be of little consequence. The research described herein evaluates audibility of differences among these techniques. As background to the description of the research it is useful to have a basic understanding of the nominal string and fret geometry and the basic physics governing vibrating strings and their frequencies of vibration, as well as of the compensation strategies to be studied.

Pythagoras is generally credited with demonstrating the relationship between the length of a taught vibrating string and its primary frequency of vibration[1]. Shortening the string increases frequency; halving the length increases frequency one octave. This general principle applies to all musical instruments with stopped strings, instruments with strings the vibrating portion of which can be shortened by pressing them to a fingerboard. Instruments with fixed frets such as guitars offer some potential advantages over instruments without them such as those of the violin family. Because fret locations are fixed, fretted instruments have the potential to provide more accurate and more consistent intonation. But fretted instruments do not provide absolute intonation accuracy by any means. The evolution of the steel string acoustic guitar includes a number of alterations intended to increase intonation accuracy. Innovation in this area continues to this day.

The vibrating lengths of the open strings of an acoustic guitar are terminated at the end of the neck by the nut and at the body end by the saddle of the bridge. The fretboard is positioned close to the strings. Distance between strings

\footnotetext{
${ }^{1}$ R.M. Mottola - Liutaio Mottola Stringed Instrument Design, Newton MA 02460 USA, rmm at LiutaioMottola dot com
} 
and the crown of the first fret is quite small and string-to-fret-crown distance increases toward the bridge end of the fretboard. This is necessary to accommodate the vibrational displacement of the open string without interference from the frets. How high the strings are above the fret tops is generally referred to as action. For steel string guitars the operational action range is quite small. Strings closer to the fretboard than would put them approximately $2.8 \mathrm{~mm}$ from the top of the $12^{\text {th }}$ fret are in danger of rattling against the frets when plucked. Action at the $12^{\text {th }}$ fret greater than approximately $3.2 \mathrm{~mm}$ would be considered by many players to be too high for comfortable playing.

The frets of a steel string acoustic guitar are typically located using an equation based on the twelfth root of two $\left(\sqrt[12]{2}\right.$ or $\left.2^{\frac{1}{12}}\right)$. This constant describes the frequency relationship among the notes of the twelve tone equal temperament tuning system (12 TET) generally in use. Equation 1 is a typical fret location equation:

$d=s-\frac{s}{2^{n / 12}}$

where $d=$ the distance of fret from the nut, $s=$ the nominal scale length (nominal vibrating length of the unfretted string), and $n=$ the fret number. Even though typical fret placement mirrors the frequency intervals of the musical tones, when the string is pressed to each fret the resulting tones will all be sharp as shown in figure 1 . This sharping is due to lengthening and thus stretching the string as it is pressed to the fret and also to bending stiffness. For typical steel guitar strings the effect of bending stiffness is small but significant. For the purposes of this introduction discussion will be limited to the effects of stretching.

The primary frequency of vibration of a theoretically perfect string (a string with no bending stiffness) was described by Mersenne[2]:

$f=\frac{1}{2 L} \sqrt{\frac{T}{\rho}}$

where $f=$ primary frequency of vibration, $L=$ vibrating length of the string, $T=$ string tension, and $\rho=$ linear mass density of the string. It is clear from eq. 2 that increasing tension will increase frequency. Tension increase is a function of the increased length of the stretched string and also of the stiffness of the material of the string (or its core if the string is wound) and of its cross sectional area. Standard mechanics references such as [3] provide equations showing the relationship between stress and strain in materials. In a reference on guitar technology[4] French includes the following equation:

$\Delta T=\frac{A E}{L} \Delta L$

where $\Delta T=$ change in tension, $A=$ cross sectional area of the string or its core, $E=$ the modulus of elasticity of the material of the string (core), $L=$ the original length of the string, and $\Delta L=$ the change in length of the string due to stretching. The change in tension and thus pitch due to the change in length of the string is apparent in figure 1. Pitch error goes up with each successive fret because the height of the string above the frets increases with each successive fret. Looking at eq. 3 it is apparent that change in tension is related to the stiffness of the material of the string and its diameter. Strings made of stiff materials like steel will result in higher tension increase for a given change in length than will those made of more elastic

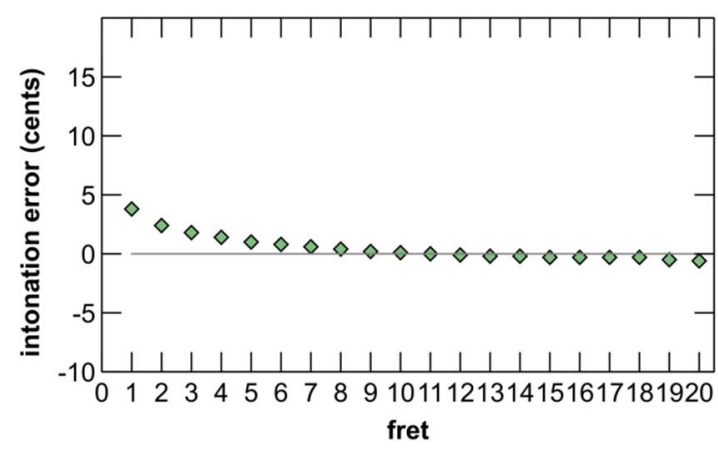

Figure 2 - Modeled intonation of typical steel string acoustic guitar string with bridge saddle compensation. The amount of compensation added for this model results in no intonation error at the $12^{\text {th }}$ fret. Overall intonation is improved but intonation remains sharp for frets $1-12$ and goes flat for frets above 12 . 
materials like nylon. Typical steel guitar strings are made of steel music wire[5] with a modulus of elasticity of approximately $200 \mathrm{GPa}$. Since each string in a string set will have a different diameter, the change in tension due to fretting for each string will be different even at the same fret.

Although it is possible to devise strategies for improving the overall intonation of the guitar based on computation it is likely that the first efforts at steel string guitar compensation were done by entirely empirical means. Compensation by changes in string length at the bridge was common among the gut strung instruments of the $19^{\text {th }}$ century[6]. The general bridge saddle compensation technique is to move the saddle back (i.e. lengthen the vibrating part of the string). From eq. 2 it can be seen that lengthening the string will lower pitch. But adding a fixed compensation length to the string will disproportionally affect the pitch at the higher frets. The general empirical technique is to iteratively move the saddle back, tune up the open string pitch (i.e. increase tension) and check intonation at the $12^{\text {th }}$ fret. Some amount of added string length and tension will result in ideal intonation for both the open string and the $12^{\text {th }}$ fretted pitch and will improve overall intonation. The pitches of fretted notes below the $12^{\text {th }}$ fret are still sharp and the pitches of fretted notes above the $12^{\text {th }}$ fret

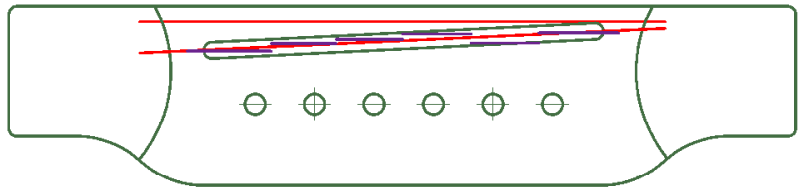

Figure 3 - Schematic representation of guitar bridge showing nominal (uncompensated) saddle location (upper red line), compensated saddle locations for each string (purple lines) and straight saddle location (lower red line). are now flat. See figure 2.

Due to differences in action and diameter of each string (core diameter for wound strings), the amount of compensation length that must be added to each string will be different. Although acoustic guitars with saddle compensation set individually for each string are popular, many instruments make use of a straight saddle. A straight saddle offers simplified construction but compensation for each string can only be approximate. Figure 3 shows a schematic representation of an acoustic guitar bridge that makes use of a straight saddle. It can be seen that the straight saddle closely approximates individual string compensation values, except for the B string (second from right). This string is a plain unwound string of relatively large diameter compared to the strings around it and so its compensation offset is out of line with the other strings. As a result a popular hybrid intonation strategy is to use a straight saddle for the other strings but to separately compensate the B string. As mentioned the historical techniques used to derive bridge saddle compensation values were empirical. Mathematical modeling makes it possible to calculate these values (see for example [7]) and online tools such as [8] are available to perform the calculations.

Although straight saddle compensation potentially offers improved intonation over no compensation at all, and individual string saddle compensation offers potentially improved intonation over straight saddle compensation, both result in intonation errors. A number of researchers have proposed additional compensation at the nut end of the string to further improve intonation. Whereas saddle compensation lengthens the string on the saddle end to flatten pitch, nut compensation shortens the string on the nut end to the same effect - the nut is moved toward the first fret. At first glance it is often not apparent how this would flatten all fretted notes. A two-step explanation is most instructive. Moving the nut toward the saddle will raise the pitch of the open string because the length of the open string is shortened. But it will have no effect on the pitches of the fretted notes, because the distances from the frets to the saddle are not affected. Now, if string tension is reduced to return the pitch of the open string to normal, that reduction in tension also lowers the pitch of all fretted notes. Figure 4 shows an application of nut compensation only to the modeled data of figure 1.

Applying both saddle and nut compensation can reduce total mathematical intonation error better than either one alone. Although lack of documentation makes the history of bridge saddle compensation somewhat vague there is ample documented research on the addition of nut compensation. The Bartolinis provided an early consideration of this at the end of an article about the dynamics of classical guitar plates[9]. Also discussed in that article was 
relocation of frets to reduce residual intonation errors. The Gilberts provided another classical guitar analysis which concluded that the addition of straight nut compensation (the same compensation applied to all strings at the nut) would provide improved intonation for that instrument[10]. The Gilberts also advocate modified fret locations. Byers, also working with nylon strung guitars, provided another analysis, and specified individual string compensation values at both saddle and nut, while leaving conventional fret locations intact[11]. Doolin suggested a pragmatic approach to steel string guitar saddle and nut compensation based on the style of music played[12]. Also working with steel string guitars, Gore and Gillet proposed a simplified approach to estimating nut compensation - calculating saddle compensation on a per string basis in the conventional manner, and then applying half of the calculated value to the saddle and the other half to the bridge[13]. These authors also proposed making use of mathematical solver tools to calculate optimal compensation at both saddle and bridge. Magliari and MacRostie proposed a method for improving intonation of steel string guitars which makes use of saddle and nut compensation and additionally relocates frets[14]. In [4] French proposed a pragmatic approach using simplified straight nut compensation and relocation of the first two frets.

Research by Varieschi and Gower also involved calculation of steel string compensation at both saddle and nut[15]. In addition to analysis and modeling to derive compensation values this research measured actual intonation, using a sonometer as an analog for a guitar. Their results indicate considerable variability in pitch for the same string/fret combination, probably due to variable fretting pressure and inadvertent string bending[16], lateral displacement of the string during fretting. It is not clear from this study if the extent of pitch variability measured on the sonometer would also be seen on the guitar. This is an issue when considering human perception of intonation

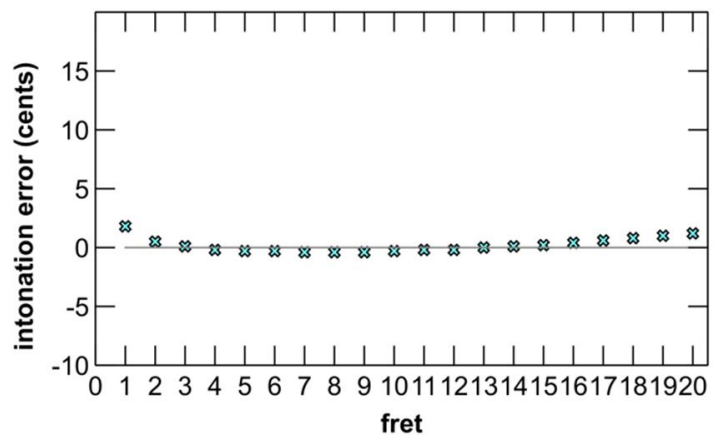

Figure 5 - Modeled intonation of typical steel string acoustic guitar string with saddle and nut compensation. The amounts of compensation added for this model results in better total intonation than can be achieved if either saddle or nut compensation were used alone. accuracy, as intonation differences below pitch variability may not be detectable. On the subject of human perception of intonation accuracy, Varieschi and Gower presented measured pitch data graphically, overlaid with human pitch discrimination range data. Although the source of these data is not explicitly cited, they correspond with those as described by [17]. It was the presentation of pitch discrimination data in this paper that suggested the value of directly studying human perception of intonation differences related to different steel string acoustic guitar compensation strategies, the subject of the experiment described below.

\section{DESCRIPTION OF THE EXPERIMENT}

Human listening evaluations have proved to be useful in differentiating the effects of physical differences in musical instruments. Work by Fritz, Cross, Moore, Woodhouse, Curtin and others[18-23] were fundamental to current understanding of human perception of physical differences in violins and in identifying correlations. The experiment described here takes a strictly psychoacoustic approach to analyzing the effect of various steel string guitar compensation strategies.

A double blind multisample intonation rating test was designed to analyze perceived intonation quality of a number of compensation strategies among experienced guitar players and guitar builders. Thirty-two subjects assented to participate in the study. Subjects were recruited at two meetings of guitar luthiers. Guitar luthiers are generally also guitarists and are familiar with issues of guitar intonation and approaches to compensation. The mean age of subjects is 50 years. Mean number of years of guitar playing experience is 26 and minimum is four years. All but three of the subjects are male. Subjects listened to short (13 second) audio clips through headphones in moderately quiet rooms and rated intonation on a scale of 1 (unacceptable intonation) to 10 (perfect intonation). The test used a randomized complete block design where each treatment was a compensation strategy. The musical sequence played in the clips was identical with the exception of intonation of some of the notes played. Each subject was presented with four trials. In each trial one audio clip representing intonation associated with one compensation strategy was played and the subject rated intonation. Compensation strategies presented were representative of straight saddle compensation, individual string saddle compensation, individual string saddle and nut compensation, and perfect intonation. The clips were presented in random order. Immediately following the four trial session another 
four trial session was presented for a total of eight trials, two each of the four compensation strategies. Subject responses are available in the supplementary document SupplementalData.xlsx.

\section{A. Selection of Compensation Strategies to be Tested}

The overview notes many different compensation strategies that have been proposed. For purposes of expediency and to limit the number of trials that each subject would be exposed to, the experiment limited the number of compensation strategies to the three listed above. These strategies are all in common use. Of the three, individual string saddle compensation is probably the most consistent from one guitar builder to another. As noted, this strategy involves setting accurate intonation at the open string and the fretted note at the $12^{\text {th }}$ fret. For this experiment intonation error values typical for individual string saddle compensation were taken from graphs appearing in [4] which were generated from data supplied by Magliari and MacRostie as supplemental to [14].

In theory straight saddle compensation can be determined by measuring or calculating individual string saddle compensation for all strings and then calculating simple linear regression to yield a straight saddle location. In practice builders making use of straight saddle compensation most often simply place the saddle at a fixed offset and angle derived from their own empirical work, or simply copy placement from available plans or from other reference material. For this experiment intonation error values typical for straight saddle compensation were measured directly from a guitar built by the author (Liutaio Mottola "Roberto" model steel string guitar).

Intonation error values typical for individual string saddle and nut compensation were also taken from [4] from research in [14]. As indicated in the overview there is no consensus on how this strategy is implemented. Selection of this implementation was primarily a matter of convenience.

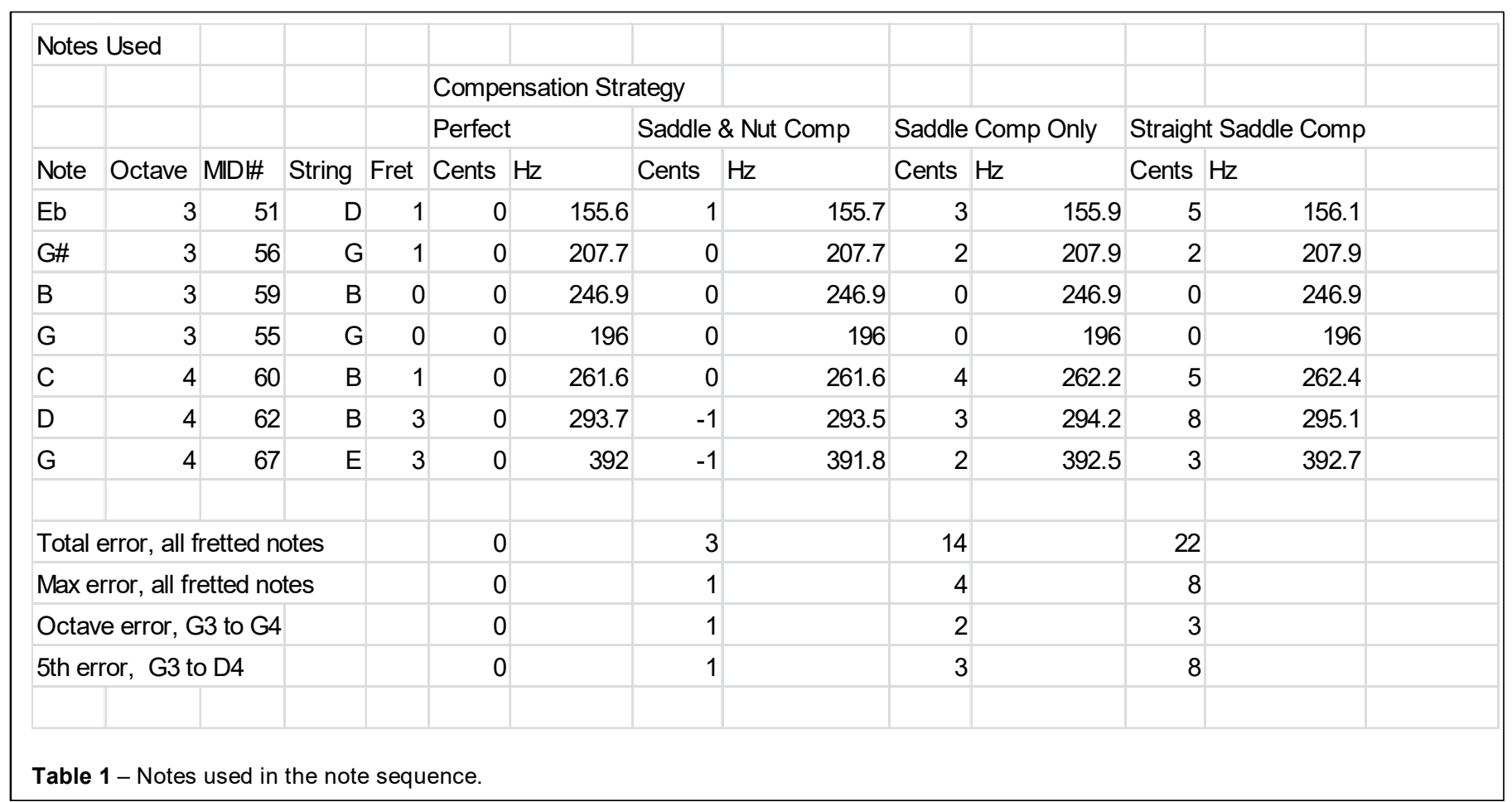

\section{B. Design of the Note Sequence}

The note sequence used in the experiment (figure 6) was devised to satisfy a number of requirements. The first of these was to present intonation errors in such a manner as to make them as readily detectable as possible. The greatest intonation error is evident on fretted notes of the first few frets for all of the compensation strategies selected for testing. Although high level intonation errors appear also on the uppermost frets for some strategies this is not an area of the guitar fretboard that gets a lot of play. For this reason the note sequence relied heavily on notes of the first three frets of the four highest pitched strings. Although the lower pitched strings generally show greater intonation errors due to their thicker core wire, human pitch discrimination is lower in the frequency range covered by these strings[17]. To aid in detection of intonation errors the note sequence presented both melodic and harmonic 
intervals. This was based on preliminary testing with three subjects, some of whom found relative pitch errors more easily detected when notes were presented sequentially and others who preferred simultaneous presentation. The presented intervals always had one open string (perfect intonation) note. The notes selected for the sequence are shown in table 1.

Intervals include the fifth and the octave, where the appearance of secondary beats may aid in identification of mistunings. Note that unison intervals are not represented in the sequence. Any unison involving an open string and a fretted note would require fretting at the fifth fret where intonation errors are minimal for all strategies tested. Ecological validity was a requirement. Although the sequence is contrived, it is musically feasible. The tempo at which the sequence is played was chosen based on feedback from the preliminary tests. Subjects preferring sequential presentation of notes also preferred these to be of relatively short duration. The opposite was true for those preferring simultaneous presentation. Although longer note duration is associated with lower detection thresholds[24], the durations used $(500 \mathrm{~ms}$ and $1000 \mathrm{~ms}$ ) in this experiment are not untypical of those used in similar studies. A final requirement was that the sequence be relatively short, in an attempt to maintain subject motivation. Duration of the sequence is less than 13 seconds.

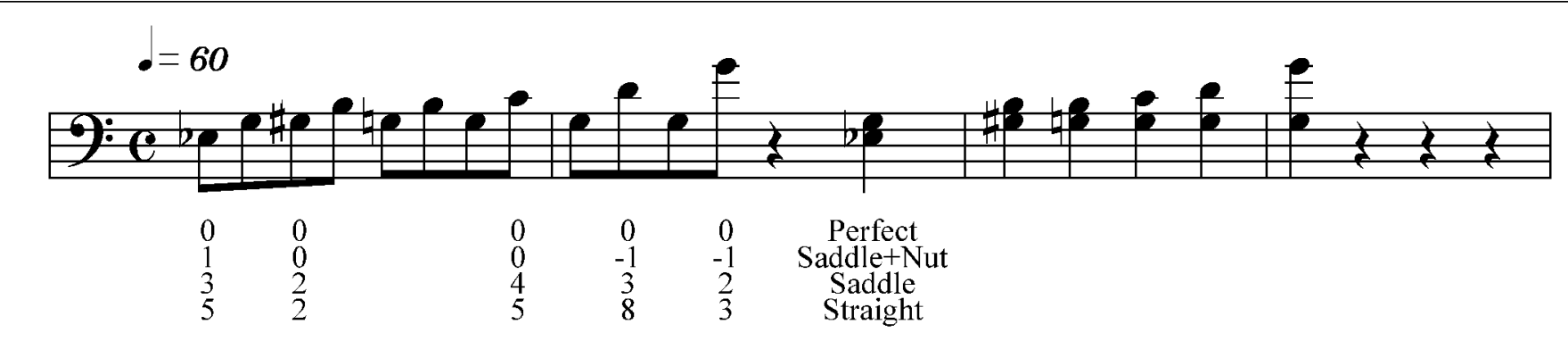

Figure 6 - Note sequence and intonation errors (in cents). Note that actual pitch is represented here (guitar notation conventionally makes use of treble clef with notes played $8 \mathrm{vb}$ ). The sequence consists of note pairs played sequentially, followed after the first rest by those same note pairs played simultaneously. Each interval (with the exception of the G-B interval, provided as a reference) is composed of one note played on an open string (and thus perfectly intonated) and one fretted note. The latter is played with the intonation error indicated for each compensation strategy tested.

\section{Preparation of the Audio Clips}

The audio clips used in the experiment needed accurate (mis)tuning of notes but otherwise needed to be identical to avoid the possibility of introducing uncontrolled performance-related independent variables such as loudness and articulation differences that could affect subject ratings. The clips were prepared as a Musical Instrument Digital Interface (MIDI)[25] sequence using the Anvil Studio sequencing software (http://anvilstudio.com/). Loudness was consistent for all notes, eighth note duration was identical and quarter note duration was identical. Note (mis)tunings were implemented using the MIDI control CC12. The sequence includes these controls before all MIDI Note On events.

The sequence was used to drive a Virtual Studio Technology (VST) sample player to generate the audio clips. The player used was Musiclab's RealGuitar (http://www.musiclab.com/products/realguitar/info.html), finger picked steel string acoustic guitar instrument, Bank 1, Prg 384. The virtual instrument responds to MIDI control CC12 as a pitch fine tuning adjustment. Recording of the clips was done using Audacity digital audio recording and editing software (http://www.audacityteam.org/), saved as uncompressed 16 bit $44.1 \mathrm{kHz}$ sampling rate .wav files. Pitch of notes in the clips was checked using AP Tuner software (http://www.aptuner.com/aptuner index.html). All sequence preparation tools were hosted on a Windows 10 PC.

\section{Testing Facility}

Audio presentation, listener rating input, and data collection were implemented using KoBoToolbox software (http://www.kobotoolbox.org/), an open source suite of tools intended for surveys and "field data collection". Although not ideally suited for the deployment of self-administered listening tests, this platform was adequate for this experiment. The software ran on an Android device (Samsung S3, which contains a 24 bit DAC for audio output) to which Sony MDR-7506 headphones were connected. The testing facility presented descriptive text about the experiment, instructions for the subject, a sample test trial so that subjects could familiarize themselves with the test and also set audio volume to a comfortable level for the test, and the eight test trials. The test also solicited subject 
age and years of guitar playing experience from each subject. The testing facility had to be initialized for each subject by a test administrator but following initialization the test was self-administered.

\section{Results AND ANALYSIS}

\section{A. General Analysis}

Subject ratings did not significantly differentiate compensation strategies. Subject ratings of the four compensation strategy treatments were analyzed by one way repeated measures ANOVA for each session. Results did not show significance for either session (session $1 p=0.596$; session $2 p=0.286$ ). Note the lower $p$-value for the second session may indicate some subject learning during the test. Follow-up t-tests were run comparing ratings for perfect intonation and the compensation treatment associated with the highest intonation errors, straight saddle compensation (two paired samples, one-tail, alpha=0.05). Results here were also not significant (session $1 \mathrm{p}=0.137$; session $2 \mathrm{p}=0.359$ ). Follow-up Bayesian estimation[26] analyses were also run, comparing these two treatments. Results indicated no credible difference for either session (session 1 difference of means 95\% HDI: -1.31, 0.472; session 2 difference of means 95\% HDI: $-0.819,1.13$ ).

\section{B. Correlating Subject Ratings and Intonation Accuracy}

Although the subject population's ratings did not identify significant perceived intonation differences among the treatments there is a possibility that some subset of subjects' ratings would do so. To identify any such candidates and for other analyses it was necessary to calculate a measure of correlation between rating and intonation accuracy. Referring to the four intonation error quantities in table 1 (total error, max error, octave error, fifth error) it can be seen that each of these are strictly monotonically increasing across compensation strategies as sorted in the table. With no a priori knowledge of how each subject would apply the rating scale (1-10, 1= unacceptable intonation, $10=$ perfect intonation) the ratings of a subject that accurately perceived all intonation differences and rated accordingly should be strictly monotonically decreasing across compensation strategies as sorted in the table. For this reason Spearman's rank correlation coefficient was calculated for each subject's ratings in each session. Session 2 results were better than those of session 1 , and range from $\rho=-1$ to $\rho=0.949$ with a mean of -0.059 .

None of the subjects' ratings met the criterion of strictly monotonically decreasing across compensation strategies. Some subjects' ratings were monotonically decreasing but not strictly so, with identical ratings given to adjacent compensation strategies. Of these most gave identical rating to more than one adjacent pair, but the two subjects with the best rating-to-intonation accuracy correlation, also in session 2, $(\rho=0.949)$ gave identical ratings to only one adjacent pair. These ratings are shown in table 2.

\begin{tabular}{|r|r|r|r|r|r|r|}
\hline Subject & Perfect Intonation & Nut and Saddle Comp. & Saddle Only Comp. & Straight Saddle Comp. \\
\hline 11 & 8 & 7 & 7 & 6 \\
\hline 21 & 7 & 7 & 5 & 4 \\
\hline
\end{tabular}

Ratings from subject 11 did not distinguish between nut and saddle compensation and saddle only compensation. Ratings from subject 21 did not distinguish between perfect intonation and nut and saddle compensation.

\section{Additional Analysis}

Age related hearing loss is well documented[27], and an association between amount of musical experience and pitch discrimination has been noted in other experiments (see for example [28]). Session 2 subject rating accuracy was compared to both subject age and years of guitar playing experience, also using Spearman's rank correlation. Both showed low correlation in this experiment (rating accuracy compared to age: $\rho=0.165$; rating accuracy compared to years of guitar playing experience: $\rho=0.059)$.

\section{Discussion AND CONCLUSION}

That the subject population did not differentiate any of the compensation treatments from perfect intonation suggests that all the treatments tested are functionally identical and all are equally effective in practice. From the perspective 
of a guitar builder the consequence of this positive result is that compensation strategy can be chosen for other reasons, such as ease or cost of implementation. Designers of computer music systems intended to closely model the acoustic guitar can likewise chose any of these treatments or perfect intonation with no perceptual penalty. Subjects in this study demonstrated a wide range of correlation between ratings of intonation and intonation accuracy. No subject ratings in this study differentiated across all treatments, but two subjects demonstrated ratings that generally tracked intonation accuracy, although they each gave the same rating to two adjacent treatments. Correlation of rating to intonation accuracy was not highly correlated with either subject age or years of guitar playing experience.

Future study in this area may benefit from larger subject populations and more sessions. A larger study and repeated testing of subjects may be able to identify a subset of the acoustic steel string guitar player population that can accurately rate intonation resulting from the compensation strategies studied. A larger study may also be able to quantify perceived differences in intonation resulting from some of the compensation strategies studied. This study originally intended to quantify difference limen/just noticeable difference (JND) for perception of intonation accuracy in both the subject population as a whole and for individual subjects. Although preliminary investigation with a small number of subjects indicated that this would generally fall within the intonation accuracies represented in the study the results of the study were quite different. Although the results may suggest that population JND would be found at a higher level of intonation accuracy than was represented in the study, future study may want to determine JND with more confidence. Note that this study made no attempt to identify documented physical or psychophysical processes involved in pitch discrimination in the study. Future work doing so may add to the knowledge on how those processes are employed in the comparison of musical tones.

\section{ACKNOWLEDGMENTS}

Many thanks to the subjects taking part in this experiment and the two organizations that provided access to those subjects, the Guild of American Luthiers and the New England Luthiers group. Samuele Carcagno provided help in selecting appropriate statistical functions and Evan Davis provided a definitive analysis of guitar intonation and cogent advice on structuring this paper. Samuele, Evan, Austin Mottola, Joseph Curtin and Christine King read and commented on early drafts of this paper. Their perspectives and commentary were invaluable. My thanks to everyone.

\section{BIBLIOGRAPHY}

1. Caleon, I. \& Ramanathan, S. (2008). From Music to Physics: The Undervalued Legacy of Pythagoras. Sci \& Educ 17: 449. Springer

2. Mersenne, Marin (1636). L'Harmonie Universelle

3. Gere, J. M., \& Timoshenko, S. (2001). Mechanics of materials Brooks. Cole, Pacific Grove, CA, 815-39.

4. French, R. M. (2012). Technology of the Guitar. Springer Science \& Business Media.

5. ASTM A228 / A228M-16, Standard Specification for Steel Wire, Music Spring Quality, ASTM International, West Conshohocken, PA, 2016, www.astm.org

6. Mottola, R.M. (2017) Was the Rule of 18 Good Enough? American Lutherie \#130 p. 52

7. Elmendorp, S. (2010). It's All About the Core or How To Estimate Compensation. American Lutherie \#104 p.56

8. Mottola, R.M. (2014) Fretted Instrument Bridge Saddle Compensation Calculator. Liutaio Mottola Lutherie Information Website. http://www.liutaiomottola.com/formulae/compensation.htm

9. Bartolini, W. and Bartolini, P. (1982). Experimental Studies of the Acoustics of Classic and Flamenco Guitars. Journal of Guitar Acoustics 6, 74

10. Gilbert, J. and Gilbert, W. (1984). Intonation and Fret Placement. Soundboard 26-27

11. Byers, G. (2006). Classical Guitar Intonation. American Lutherie \#47 p.34

12. Doolin, M. (2007). Intonation in the Real World. American Lutherie \#92 p.26

13. Gore, T. and Gillet, G. (2011) Contemporary Acoustic Guitar Design and Build 
14. Magliari, G. and MacRostie, D. (2013). Beyond the Rule of 18: Intonation For the 21st Century. American Lutherie \#116 p.6

15. Varieschi, G. U. and Gower, C. M. (2010). Intonation and compensation of fretted string instruments. American Journal of Physics, 78(1), 47-55.

16. Mottola, R. M. (2014). Guitar Fretboard Camber and Action in the Context of String Bending. Savart Journal, 1(4).

17. Shower, E. G. and Biddulph, R. (1931). Differential pitch sensitivity of the ear. The Journal of the Acoustical Society of America, 3(2A), 275-287.

18. Fritz, C., Cross, I., Smith, E., Weaver, K., Petersen, U., Woodhouse, J., \& Moore, B. C. J. (2006, August). Perceptual correlates of violin acoustics. In Proc. of 9th Int. Conf. on Music Perception and Cognition, Bologna, Italy.

19. Fritz, C., Cross, I., Moore, B. C., \& Woodhouse, J. (2007). Perceptual thresholds for detecting modifications applied to the acoustical properties of a violin. The Journal of the Acoustical Society of America, 122(6), 36403650.

20. Fritz, C., Woodhouse, J., Cheng, F. P. H., Cross, I., Blackwell, A. F., \& Moore, B. C. (2010). Perceptual studies of violin body damping and vibrato. The Journal of the Acoustical Society of America, 127(1), 513-524.

21. Fritz, C., Curtin, J., Poitevineau, J., Morrel-Samuels, P., \& Tao, F. C. (2012). Player preferences among new and old violins. Proceedings of the National Academy of Sciences, 109(3), 760-763.

22. Fritz, C., Curtin, J., Poitevineau, J., Borsarello, H., Tao, F. C., \& Ghasarossian, T. (2014). Soloist evaluations of six Old Italian and six new violins. Proceedings of the National Academy of Sciences, 111(20), 7224-7229.

23. Fritz, C., Curtin, J., Poitevineau, J., \& Tao, F. C. (2017). Listener evaluations of new and Old Italian violins. Proceedings of the National Academy of Sciences, 114(21), 5395-5400.

24. Feeney, M. P. (1997). Dichotic beats of mistuned consonances. The Journal of the Acoustical Society of America, 102(4), 2333-2342.

25. MIDI Association. The Official MIDI Specifications. https://www.midi.org/specifications

26. Kruschke, J. K. (2013). Bayesian estimation supersedes the t test. Journal of Experimental Psychology: General, 142(2), 573.

27. Frisina, R. D. (2009). Age-related hearing loss. Annals of the New York Academy of Sciences, 1170(1), 708-717.

28. Kishon-Rabin, L., Amir, O., Vexler, Y., \& Zaltz, Y. (2001). Pitch discrimination: Are professional musicians better than non-musicians? Journal of basic and clinical physiology and pharmacology, 12(2), 125-144. 